\title{
ENXERTIA DE MESA DE Passiflora edulis Sims f. flavicarpa Deg. SOBRE Passiflora alata Curtis, EM AMBIENTE DE NEBULIZAÇÃO INTERMITENTE ${ }^{1}$
}

\author{
FLÁVIA MATOS SILVA², LUIZ DE SOUZA CORRÊA³, APARECIDA CONCEIÇÃO BOLIANI ${ }^{4}$, \\ PEDRO CÉSAR DOS SANTOS
}

\begin{abstract}
RESUMO - O maracujazeiro é uma cultura típica de países tropicais, sendo estes responsáveis por cerca de 90\% da produção mundial. A propagação vegetativa do maracujazeiro permite a obtenção de pomares uniformes, bem como de porta-enxertos altamente produtivos ou resistentes a doenças. O experimento teve como objetivo verificar a viabilidade da enxertia de mesa do maracujazeiro-amarelo (Passiflora edulis Sims f. flavicarpa Deg.) sobre o maracujazeiro-doce (Passiflora alata Curtis), conduzido em telado com 50\% de sombreamento, dotado de sistema de nebulização intermitente. Foi conduzido de agosto de 2001 a maio de 2002, na Fazenda de Ensino e Pesquisa da UNESP - Câmpus de Ilha Solteira, localizada no município de Selvíria-MS. O delineamento experimental utilizado foi blocos ao acaso, esquema de parcelas subdivididas no tempo, totalizando quatro tratamentos (T1 - enxertia tipo fenda cheia, com o garfo do ponteiro dos ramos; T2 - enxertia tipo inglês simples, com o garfo do ponteiro dos ramos; T3 - enxertia tipo fenda cheia, com o garfo da parte mediana dos ramos; T4 - enxertia tipo inglês simples, com o garfo da parte mediana dos ramos), cinco repetições e 25 estacas enxertadas por parcela. Foram avaliados: a) porcentagem de sobrevivência das estacas enxertadas; b) porcentagem de estacas enxertadas enraizadas; c) número de brotos emitidos por estaca enxertada; d) número de folhas emitidas por estaca enxertada; e) massa da matéria seca da raiz, caule, folha e planta. Com base nos resultados, pode-se concluir que: a técnica de enxertia de mesa, quando aplicada ao maracujazeiro, mostrou-se viável, com excelente porcentagem de sobrevivência e enraizamento.
\end{abstract}

Termos para indexação: maracujazeiro, propagação.

\section{BENCH GRAFT OF Passiflora edulis Sims f. flavicarpa Deg. ON Passiflora alata Curtis, IN INTERMITTENT MISTY} ATMOSPHERE

\begin{abstract}
The passion fruit plant is a typical culture of tropical countries, which are responsible for about $90 \%$ of the world production. The vegetative propagation of the passion fruit plant allows the obtainment of uniform orchards, as well as of rootstocks highly productive or resistant to diseases. The experiment has had as objective to verify the viability of the bench graft of yellow passion fruit plant (Passiflora edulis Sims f. flavicarpa Deg.) on to the sweet passion fruit plant (Passiflora alata Curtis), in greenhouse with $50 \%$ of light reduction, endowed with system of intermittent misty. It was conducted from August 2001 to May 2002 at Experimental Station of UNESP - Campus of Ilha Solteira, Selvíria - MS/Brazil. The used experimental design was randomized blocks, totalizing four treatments (T1 - cleft grafting, with graft of the terminal part of the branches; T2 - whip grafting, with graft of the terminal part of the branches; T3 - cleft grafting, with graft of the medium part of the branches; T4 - whip grafting, with graft of the medium part of the branches), with five repetitions and 25 cuttings grafted for portion. The evaluated variabilities were: a) percentage of survival of the grafted cuttings; b) percentage of rooted grafted cuttings; c) number of emitted sprouts by grafted cutting; d) number of leaves emitted by grafted cutting; e) dry matter mass of the root, stem, leaf and plant. It can be concluded that: the technique of bench graft applied to the passion fruit plant was shown viable, with excellent survival and rooting percentages.
\end{abstract}

Index terms: passion fruit plant, propagation.

\section{INTRODUÇÃO}

O maracujazeiro pertence à Família Passifloraceae, gênero Passiflora, o qual é constituído de 530 espécies, das quais 150 são nativas do Brasil e, destas, 60 produzem frutos comestíveis (Schultz, 1953). É uma cultura típica de países tropicais, que são responsáveis por cerca de $90 \%$ da produção mundial. O Brasil é o maior produtor de maracujá, seguido pelo Peru, Venezuela, África do Sul, Sri Lanka e Austrália. No ano de 2000, a área colhida no Brasil foi de 33,4 mil hectares, destacando-se os Estados da Bahia, São Paulo, Sergipe, Minas Gerais, Goiás, Espírito Santo, Ceará, Pará, Rio de Janeiro e Alagoas (Agrianual, 2003).

As espécies mais cultivadas no Brasil e em toda a América Tropical, para a obtenção de frutos para consumo in natura ou para fins de industrialização, são: $P$. edulis f. flavicarpa Deg., P.edulis f. edulis Sims, $P$. alata, $P$. quadrangularis, $P$. macrocarpa, $P$. caerula $e P$. laurifolia. Mas a espécie $P$. edulis f. flavicarpa Deg. (maracujazeiroamarelo) destaca-se como a mais cultivada no Brasil e no Hawaí.

A espécie Passiflora alata Curtis (maracujazeiro-doce) está mostrando ser uma opção apropriada para ocupar não somente o mercado interno, onde seus frutos atingem preços elevados, como também o mercado externo de frutos in natura (Vasconcellos et al., 1993), além de ser considerada resistente à fusariose, juntamente com as espécies $P$. quadrangularis e $P$. macrocarpa.

O maracujazeiro pode ser propagado por sementes, bem como por estaquia e enxertia (Ruggiero \& Corrêa, 1980). No entanto, sua propagação, em escala comercial, é realizada por via sexual, mas a elevada heterozigosidade existente determina alta variabilidade, decorrendo deste fato a falta de uniformidade dos pomares. Plantas-matrizes com características desejáveis, como elevada produtividade e frutos com teores elevados de suco e de sólidos solúveis totais, podem ser propagadas através de estaquia e enxertia. Entretanto, deve-se levar em consideração que o maracujazeiro é uma planta auto-incompatível (Bruckner et al., 1995), e por isso, no caso da propagação vegetativa, fazse necessária a utilização de material de várias plantas, para viabilizar a produção de frutos.

Lima (1999) verificou que a estaquia de maracujazeiro, com nebulização intermitente, tem dado excelentes resultados quanto à porcentagem de enraizamento.

No que diz respeito ao processo de enxertia, Ruggiero (1991)

\footnotetext{
${ }^{1}$ (Trabalho 083/2004). Recebido: 30/01/2004. Aceito para publicação: 21/01/2005.

${ }^{2}$ Bióloga, Mestre em Agronomia - FEIS/UNESP, Passeio Londrina, 409, CEP:15385-000, Ilha Solteira, SP, Brasil, (18) 3742-2286, flaviaisa@ click21.com.br.

${ }^{3}$ Prof. Titular da FEIS/UNESP, Depto. Fitotecnia, Tecnologia de Alimentos e Sócio-Economia, Av. Brasil, 56, CEP:15385-000, Ilha Solteira, SP, Brasil, (18) 37431248.1correa@feis.unesp.br.

${ }^{4}$ Prof. Adjunto da FEIS/UNESP, Depto. Fitotecnia, Tecnologia de Alimentos e Sócio-Economia, Av. Brasil, 56, CEP:15385-000, Ilha Solteira, SP, Brasil, (18) 37431243. boliani@agr.feis.unesp.br.

${ }^{5}$ Prof. Adjunto da FEIS/ UNESP, Depto. Fitotecnia, Tecnologia de Alimentos e Sócio-Economia, Av. Brasil, 56, CEP:15385-000, Ilha Solteira, SP, Brasil, (18) 37431252.santospc@agr.feis.unesp.br.
} 
TABELA 1 - Valores de sobrevivência, número de brotos e número de folhas emitidas por estaca de maracujazeiro-amarelo enxertada sobre o maracujazeiro-doce, em diferentes épocas, após a enxertia de mesa. Selvíria-MS, 2001.

\begin{tabular}{|c|c|c|c|c|c|}
\hline \multirow[b]{3}{*}{ Tratamentos } & \multicolumn{5}{|c|}{ Sobrevivência (\%) ${ }^{1}$} \\
\hline & \multicolumn{5}{|c|}{ Dias após a enxertia } \\
\hline & 10 & 20 & 30 & 40 & 50 \\
\hline 1- Fenda cheia, garfo do ponteiro & $100 \mathrm{a}$ & $98,4 \mathrm{a}$ & $96,8 \mathrm{a}$ & $93,6 \mathrm{a}$ & $86,4 \mathrm{a}$ \\
\hline 2- Inglês simples, garfo do ponteiro & $100 \mathrm{a}$ & $97,6 \mathrm{a}$ & $91,2 \mathrm{a}$ & $88,0 \mathrm{a}$ & $78,4 \mathrm{a}$ \\
\hline 3- Fenda cheia, garfo da parte mediana & $100 \mathrm{a}$ & $99,2 \mathrm{a}$ & 98,4 a & $97,6 \mathrm{a}$ & $96,8 \mathrm{a}$ \\
\hline 4- Inglês simples, garfo da parte mediana & $100 \mathrm{a}$ & $98,4 \mathrm{a}$ & $93,6 \mathrm{a}$ & $90,4 \mathrm{a}$ & $86,4 \mathrm{a}$ \\
\hline \multicolumn{6}{|l|}{$\mathrm{CV}=6,473 \%$} \\
\hline & \multicolumn{5}{|c|}{ Número de brotos emitidos por estaca enxertada ${ }^{1}$} \\
\hline 1- Fenda cheia, garfo do ponteiro & $0,00 \mathrm{a}$ & $0,45 \mathrm{a}$ & $0,72 \mathrm{a}$ & $0,91 \mathrm{a}$ & $1,09 \mathrm{a}$ \\
\hline 2- Inglês simples, garfo do ponteiro & $0,00 \mathrm{a}$ & $0,39 \mathrm{a}$ & $0,67 \mathrm{a}$ & $0,82 \mathrm{a}$ & $1,06 \mathrm{a}$ \\
\hline 3- Fenda cheia, garfo da parte me diana & $0,00 \mathrm{a}$ & $0,60 \mathrm{a}$ & $0,81 \mathrm{a}$ & $1,07 \mathrm{a}$ & $1,26 \mathrm{a}$ \\
\hline 4- Inglês simples, garfo da parte mediana & $0,00 \mathrm{a}$ & $0,53 \mathrm{a}$ & $0,84 \mathrm{a}$ & $0,96 \mathrm{a}$ & $1,13 \mathrm{a}$ \\
\hline \multicolumn{6}{|l|}{$\mathrm{CV}=3,950 \%$} \\
\hline & \multicolumn{5}{|c|}{ Número de folhas emitidas por estaca enxertada ${ }^{1}$} \\
\hline 1- Fenda cheia, garfo do ponteiro & $0,00 \mathrm{a}$ & $0,00 \mathrm{a}$ & $0,00 \mathrm{a}$ & $1,08 \mathrm{a}$ & $2,63 \mathrm{a}$ \\
\hline 2- Inglês simples, garfo do ponteiro & $0,00 \mathrm{a}$ & $0,00 \mathrm{a}$ & $0,00 \mathrm{a}$ & $0,64 \mathrm{~b}$ & $1,85 \mathrm{~b}$ \\
\hline 3- Fenda cheia, garfo da parte mediana & $0,00 \mathrm{a}$ & $0,00 \mathrm{a}$ & $0,00 \mathrm{a}$ & $1,03 \mathrm{ab}$ & $2,71 \mathrm{a}$ \\
\hline 4- Inglês simples, garfo da parte mediana & $0,00 \mathrm{a}$ & $0,00 \mathrm{a}$ & $0,00 \mathrm{a}$ & $0,99 \mathrm{ab}$ & $1,94 \mathrm{~b}$ \\
\hline
\end{tabular}

$\mathrm{CV}=8,085 \%$

${ }^{1}$ Médias seguidas de mesma letra, na coluna, não diferem entre si, a 5\% de probabilidade, pelo teste de Tukey.

afirma que os tipos mais usados são: fenda cheia e inglês simples, com pegamento superior a 90\%, em ambos os processos. Ruggiero \& Corrêa (1980) afirmam que deve ser realizada no início da brotação primaveril.

A enxertia poderá ter grande importância para a cultura, na solução de problemas relativos a pragas e doenças. Menezes et al. (1994) trabalharam, em Jaboticabal - SP, com enxertia de maracujazeiro-amarelo sobre espécies tidas como resistentes à murcha de fusarium. Constataram que havia diferença quanto ao pegamento entre as espécies estudadas e que, utilizando porta-enxerto de maracujazeiro-doce, obtiveram $93,7 \%$ de pegamento.

Oliveira (1980) estudou o maracujazeiro-amarelo enxertado sobre Passiflora alata, P. macrocarpa e P. quadrangularis, cultivados em solo contaminado com Fusarium oxysporum f. passiflorae. O melhor resultado foi obtido quando se utilizou P. alata como porta-enxerto.

Em outras culturas, como a videira (Albuquerque \& Albuquerque, 1984; Pereira et al., 1998), tem-se utilizado a enxertia de mesa por garfagem ou borbulhia, com excelente pegamento. Essa técnica consiste em justapor um garfo ou borbulha em uma estaca já enraizada, ou que será colocada em substrato para enraizamento e desenvolvimento da parte aérea, e tem, entre outras vantagens, a de produzir mudas mais rapidamente.

O objetivo do trabalho foi verificar a viabilidade da enxertia de mesa de maracujazeiro-amarelo (Passiflora edulis Sims f. flavicarpa Deg.) sobre o maracujazeiro-doce (Passiflora alata Curtis), conduzido em telado com sistema de nebulização intermitente.

\section{MATERIAL E MÉTODOS}

O experimento foi instalado, no campo experimental da UNESP - Câmpus de Ilha Solteira, localizado no município de Selvíria - MS.

O delineamento experimental utilizado foi blocos ao acaso, no esquema de parcelas subdivididas no tempo, segundo Stork et al. (1995), sendo constituído de quatro tratamentos ( 1 - enxertia tipo fenda cheia, com garfo do ponteiro dos ramos; 2 - enxertia tipo inglês simples, com garfo do ponteiro dos ramos; 3 - enxertia tipo fenda cheia, com garfo da parte mediana dos ramos; 4 - enxertia tipo inglês simples, com garfo da parte mediana dos ramos), cinco repetições e 25 estacas enxertadas por parcela.

Como porta-enxerto, foi utilizado o maracujazeiro-doce (P. alata Curtis) e como enxerto, o maracujazeiro-amarelo (P. edulis f. flavicarpa Deg.).
As estacas utilizadas como porta-enxerto e os garfos foram retirados de plantas de 1,5 ano de idade, e colocados com a base em água, no mesmo dia da coleta (13-08-01). As estacas destinadas aos garfos foram retiradas das partes mediana e apical dos ramos, e somente da parte mediana para o porta-enxerto. Cada uma continha dois nós e uma folha. Procedeu-se, então, à garfagem em fenda cheia ou inglês simples com auxílio da fita de enxertia. As bases das estacas enxertadas $(3 \mathrm{~cm}$ ) foram tratadas por imersão em Benlate (benomyl) na dose de $65 \mathrm{~g} /$ 100 litros de água, durante um minuto, e posteriormente transferidas para o substrato.

O enraizamento das estacas enxertadas foi realizado em caixas plásticas contendo vermiculita média expandida, dentro de um telado com 50\% de sombreamento, sob nebulização intermitente (40/7 segundos), durante os 50 primeiros dias. A cada dez dias, foram avaliados a porcentagem de sobrevivência das estacas e o número de brotos e de folhas emitidas por estaca enxertada. Aos 50 dias, foram avaliadas a porcentagem de estacas enxertadas enraizadas e a massa da matéria seca da raiz, do caule, da folha e da planta.

Os dados coletados foram transformados em arco-seno da raiz de $\mathrm{x} / 100$, para os dados referentes à porcentagem, e em raiz de $\mathrm{x}+0,5$, para os dados referentes às contagens, sendo que os demais dados, relacionados à massa da matéria seca (da raiz, do caule, da folha e da planta), não sofreram transformação (Banzato \& Kronka, 1989). Todos os dados foram submetidos à análise de variância, e as médias foram comparadas pelo teste de Tukey, a 5\% de probabilidade. Para o fator época, efetuou-se análise de regressão polinomial com os dados originais, para as variáveis: porcentagem de sobrevivência, número de brotos e número de folhas emitidos por estaca enxertada.

\section{RESULTADOS E DISCUSSÃO}

Pela Tabela 1, verifica-se que não houve diferença estatística da porcentagem de sobrevivência de estacas de maracujazeiro-amarelo enxertadas sobre maracujazeiro-doce entre os tratamentos, em nenhuma das épocas avaliadas.

A sobrevivência das estacas enxertadas variou em função do tempo, cujos dados se ajustaram a uma função quadrática com queda acentuando-se a partir dos 30 dias, conforme se verifica na Figura 1.

Observa-se, ainda pela Tabela 1, que em relação aos dados referentes ao número médio de brotos emitidos por estaca enxertada, não houve diferença estatística significativa entre os tratamentos, em 
qualquer época de avaliação, evidenciando que não houve efeito do tipo de enxertia sobre a brotação. O número de brotos por estaca teve um comportamento semelhante nos quatro tratamentos, ou seja, aumentou com o tempo, conforme se verifica na Figura 2. Observa-se que aos 50 dias havia, em média, 1,2 broto por estaca enxertada.

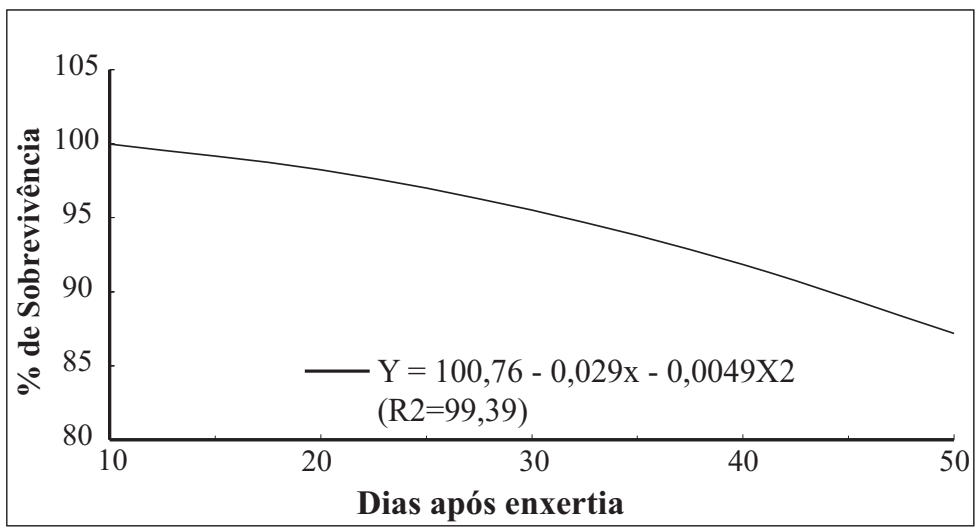

FIGURA 1 - Porcentagem de sobrevivência de estacas de maracujazeiroamarelo enxertadas sobre o maracujazeiro-doce, em diferentes épocas, após a enxertia de mesa. Selvíria - MS, 2001

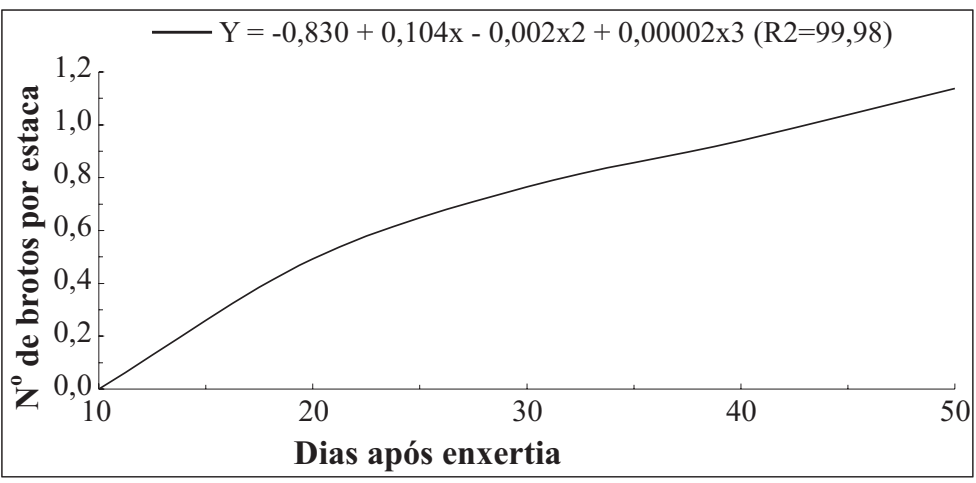

FIGURA 2 - Número de brotos emitidos por estaca de maracujazeiroamarelo enxertada sobre o maracujazeiro-doce, em diferentes épocas, após a enxertia de mesa. Selvíria-MS, 2001.

Em relação ao número médio de folhas emitidas por estaca enxertada, verifica-se que não houve emissão de folhas durante os primeiros 30 dias. Aos 50 dias, o melhor resultado foi alcançado quando se utilizou enxertia tipo fenda cheia, concordando com Corrêa et al. (1979), que estudaram a enxertia de maracujazeiro-amarelo sobre amarelo, em mudas formadas por sementes. Assim, nas condições do experimento, a enxertia por fenda cheia pareceu permitir melhor contato entre o enxerto e porta-enxerto, facilitando o desenvolvimento do conjunto. Verifica-se, pela Figura 3, que, em relação aos dados referentes à mesma avaliação (número de folhas emitidas por estaca enxertada), houve resposta diferenciada dos tratamentos em função do tempo, ou seja, constatouse interação tratamento $X$ época. Na mesma figura, pode-se perceber que até os 30 dias não houve o aparecimento de folhas. A partir daí, constatou-se aumento no número de folhas por estaca, sendo que os tratamentos de fenda cheia (garfo do ponteiro e garfo da parte mediana) tiveram melhor desempenho, atingindo, em média, 2,7 folhas por estaca.

Pela Tabela 2, verifica-se que a porcentagem de estacas enxertadas enraizadas variou de 72 a $85,6 \%$, e não houve diferença estatística significativa entre os tratamentos. Tais resultados estão de acordo com os obtidos por Menezes et al. (1994), Ruggiero \& Oliveira (1998) e Lima et al. (1999), com enxertia (garfagem) convencional (portaenxertos obtidos de sementes). Com relação aos tipos de enxertia, os resultados estão de acordo com os obtidos por Lima et al. (1999), que concluíram que a garfagem tipo fenda cheia não diferiu da garfagem tipo inglês simples.

Quanto à porcentagem de pegamento da enxertia de mesa, os resultados obtidos estão próximos aos encontrados por Corrêa et al. (1979) com enxertia de maracujazeiro-amarelo sobre amarelo, com mudas formadas por sementes, que obtiveram $95 \%$ de pegamento para o tipo fenda cheia e $50 \%$ para o tipo inglês simples. Resultados semelhantes foram obtidos por Menezes et al. (1994), em Jaboticabal, com enxertia de maracujazeiro-amarelo sobre maracujazeiro-doce obtido de sementes, que resultou em $93,7 \%$ de pegamento.

Considerando os dados obtidos por Menezes et al. (1994) e Corrêa et al. (1979), pode-se dizer que os resultados do presente trabalho foram bons em termos de enraizamento e pegamento, e que na fase inicial de desenvolvimento não há evidências de incompatibilidade entre o porta-enxerto (maracujazeiro-doce) e o enxerto (maracujazeiro-amarelo).

Verifica-se que a enxertia de mesa é viável para a combinação testada, principalmente quando se utiliza a garfagem tipo fenda cheia, assim como foi constatado por Albuquerque \& Albuquerque (1984), em videira

Pela massa da matéria seca, verifica-se que, para raiz, não houve diferença estatística significativa entre os tratamentos. Porém para caule, folhas e planta (total), as diferenças apareceram, sendo que o maior valor numérico de massa da matéria seca sempre foi obtido com o tratamento 3 (enxertia por fenda cheia com garfo da parte mediana dos ramos). Desta forma, mais uma vez, nota-se que a enxertia de mesa pelo tipo fenda cheia parece permitir melhor desenvolvimento inicial das mudas.

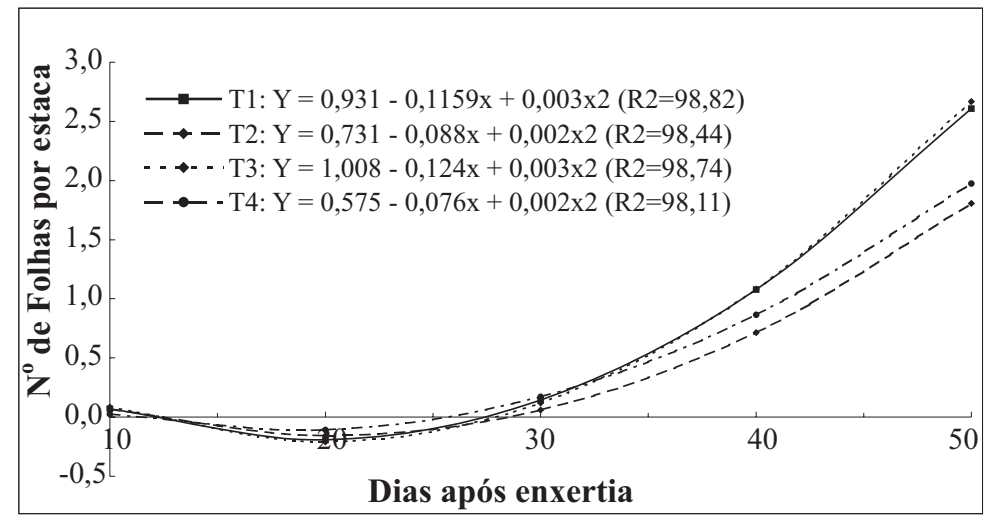

FIGURA 3 - Número de folhas emitidas por estaca de maracujazeiro-amarelo enxertada sobre o maracujazeiro-doce, em diferentes épocas, após a enxertia de mesa. Selvíria-MS, 2001.

TABELA 2 - Porcentagem média de estacas enxertadas enraizadas e massa da matéria seca (g) da raiz, do caule, da folha e da planta (total), por estaca de maracujazeiro-amarelo enxertada sobre o maracujazeiro-doce, aos 50 dias após a enxertia de mesa. Selvíria - MS, 2001.

\begin{tabular}{|c|c|c|c|c|c|}
\hline \multirow[t]{2}{*}{ Tratamentos } & \multirow{2}{*}{$\begin{array}{c}\text { Estacas enxertadas } \\
\text { enraizadas }(\%)^{1}\end{array}$} & \multicolumn{4}{|c|}{ Massa da matéria seca $(\mathrm{g})^{1}$} \\
\hline & & Raiz & Caule & Folha & Planta \\
\hline 1 - Fenda cheia, garfo do ponteiro & $82,4 \mathrm{a}$ & $0,11 \mathrm{a}$ & $1,87 \mathrm{~b}$ & $0,63 \mathrm{ab}$ & $2,53 \mathrm{ab}$ \\
\hline 2 - Inglês simples, garfo do ponteiro & $72,0 \mathrm{a}$ & $0,11 \mathrm{a}$ & $1,74 \mathrm{~b}$ & $0,50 \mathrm{ab}$ & $2,35 \mathrm{~b}$ \\
\hline 3 - Fenda cheia, garfo da parte mediana & $85,6 \mathrm{a}$ & $0,12 \mathrm{a}$ & $2,42 \mathrm{a}$ & $0,80 \mathrm{a}$ & $3,33 \mathrm{a}$ \\
\hline 4 - Inglês simples, garfo da parte mediana & $73,6 \mathrm{a}$ & $0,07 \mathrm{a}$ & $2,22 \mathrm{ab}$ & $0,33 \mathrm{~b}$ & $2,62 \mathrm{ab}$ \\
\hline $\mathrm{CV}(\%)$ & 14,71 & 31,88 & 13,08 & 38,5 & 16,25 \\
\hline
\end{tabular}

${ }^{1}$ Médias seguidas de mesma letra, na coluna, não diferem entre si, a 5\% de probabilidade, pelo teste de Tukey. 


\section{CONCLUSÕES}

Nas condições em que foi conduzido o trabalho, pode-se concluir que: a enxertia de mesa mostrou-se viável, com excelente porcentagem de sobrevivência $(96,8)$ e enraizamento das estacas $(85,6)$.

\section{REFERÊNCIAS}

AGRIANUAL 2003: Anuário da agricultura brasileira. Maracujá. São Paulo: FNP Consultoria e Comércio, 2002. p.399-405

ALBUQUERQUE, T.C.S.; ALBUQUERQUE, J.A.S. de. Enxertia de mesa em videira no Submédio São Francisco. Petrolina-PE: EMBRAPACPATSA, 1984. 6p. (Comunicado Técnico, 12).

BANZATO, D.A.; KRONKA, S. do N. Experimentação agrícola. Jaboticabal: Faculdade de Ciências Agrárias e Veterinárias, UNESP, 1989.247p.

BRUCKNER, C.H.; CASALI, V.W.D.; REGAZZI, A.J.; SILVA, A.M. Selfincompatibility in passion fruit (Pasiflora edulis Sims). Acta Horticulturae, Wageningen, n. 370, p. 45-57, 1995.

CORRÊA, L. de S.; RUGGIERO, C.; OLIVEIRA, J.C. Propagation of yellow passion fruit by graftage. Proceendings Tropical Region A.S.H.S., Jaboticabal, v. 23, p.149-150, 1979.

LIMA, A. de A.; CALDAS, R.C.; CUNHA, M.A.P. da.; FILHO, H.P.S. Avaliação de porta-enxertos e tipos de enxertia para o maracujáamarelo. Revista Brasileira de Fruticultura, Jaboticabal, v. 21, n. 3, p.318-321, 1999.

LIMA, E.S. Efeitos de épocas de estaquia, diferentes tipos de estaca e concentrações de ácido indolbutír co (AIB) no enraizamento de estacas de maracujazeiro-doce (Passiflora alata Dryand). 1999. 27 f. Monografia (Trabalho de graduação em Agronomia) - Faculdade de Engenharia do Campus de Ilha Solteira, Ilha Solteira, 1999.
MENEZES, J.M.T.; OLIVEIRA, J.C. de.; RUGGIERO, C.; BANZATTO, D.A. Avaliação da taxa de pegamento de enxertos de maracujá-amarelo sobre espécies tolerantes à "morte prematura de plantas". Científica, São Paulo, v.1, n.22, p.95-104, 1994.

OLIVEIRA, J.C. Melhoramento genético do maracujazeiro. In: RUGGIERO, C. Cultura do maracujazeiro. Jaboticabal: Faculdade de Ciências Agrárias e Veterinárias, UNESP, 1980. p.115-130.

PEREIRA, G.E.; REGINA, M.A.; SOUZA, C.R.; PEREIRA, A. F. Enxertia de mesa com forçagem na propagação de videiras oriundas de regiões tropicais. In: CONGRESSO BRASILEIRODE FRUTICULTURA, 15., 1998, Poços de Caldas. Resumos... Poços de Caldas: SBF, 1998. p.745.

RUGGIERO, C.; CORRÊA, L. S. Implantação da cultura e propagação. In: RUGGIERO, C. Cultura do maracujazeiro. Jaboticabal: Faculdade de Ciências Agrárias e Veterinárias, UNESP, 1980. p.23-31.

RUGGIERO, C. Enxertia do maracujazeiro. In: SÃO JOJÉ,A. R., FERREIRA, F.R.; VAZ, R. L. A cultura do maracujá no Brasil. Jaboticabal: FUNEP, 1991.p.43-60.

RUGGIERO, C.; OLIVEIRA, J.C. de. Enxertia do maracujazeiro. In: SIMPÓSIO SOBREACULTURADO MARACUJAZEIRO, 5., 1998, Jaboticabal. Anais... Jaboticabal: UNESP/FCAV, 1998. p.70-92.

SCHULTZ, A. Botânica sistemática. Porto Alegre: Globo, 1953. 562p.

STORK, L. et al. Experimentos fatoriais: modelos de análise pelos pacotes SAS, SAEG e SOC. Santa Maria: Universidade Federal de Santa Maria - Centro de Ciências Rurais, 1995. 53p.

VASCONCELLOS, M.A. da S.; CEREDA, E.; ANDRADE, J.M. de B.; FILHO, J.U.T.B. Desenvolvimento de frutos de maracujazeiro "Doce" (Passiflora alata Dryand), nas condições de Botucatu - SP. Revista Brasileira de Fruticultura, Cruz das Almas, v. 15, p.153-158, 1993. 